\title{
A novel surgical method for total nail ablation: Use of triple flap technique
}

\author{
Berna Aksoy ${ }^{1,2^{*}}$ \\ ${ }^{1}$ Bahcesehir University, Faculty of Medicine, Dermatology Department, Istanbul, Turkey \\ ${ }^{2}$ VM Medicalpark Hospital, Dermatology Clinic, Kocaeli, Turkey
}

\begin{abstract}
Total nail ablation for the treatment of onychodystrophies can be performed by using chemicals, surgical resection or laser ablation of nail matrix. A female patient with bilateral severe onychodystrophy as a result of inadvertent previous nail surgeries was treated surgically. Proximal complete nail matrix resection and distal 10 mm wide transverse strip partial nail bed resection were performed. A $5 \mathrm{~mm}$ wide transverse strip of nail bed was left intact proximally. Proximal nail matrix defect was closed by using the skin of proximal nail fold. The distal nail bed defect was reconstructed by using triple flap technique which was composed of a main central advancement flap containing ventral toe skin and two side flaps containing nail fold skin. The patient healed without any problem and her nail problem was treated successfully. The cosmetic appearance of her first toes was acceptable. Surgical nail ablation followed by volar skin coverage of dorsal surface of the distal phalanx bone by using triple flap technique is an effective surgical treatment method for the correction of advanced nail plate deformities requiring total nail ablation.
\end{abstract}

Keywords: ablation; dystrophy; flap; nail; onychodystrophy; surgery; treatment

Citation: Aksoy B. A novel surgical method for total nail ablation: Use of triple flap technique. J Surg Dermatol 2021; 6(2): 167; http://dx.doi.org/10.18282/jsd.v6.i2.167.

*Correspondence to: Berna Aksoy, VM Medicalpark Hastanesi, Ovacik mah, D-100 Karayolu Ustu, No: 36, Basiskele, Kocaeli, Turkey; bmaksoy@mynet.com, bernaaaksoy@gmail.com

Received: $8^{\text {th }}$ April 2021; Accepted: $1^{\text {st }}$ July 2021; Published Online: $7^{\text {th }}$ July 2021

\section{Introduction}

When a nail becomes severely deformed permanent total ablation of the nail is the best choice of treatment ${ }^{[1]}$. Here we describe a case with severe bilateral great toe onychodystrophy treated by total surgical nail matrix excision followed by plantar skin coverage of dorsal aspect of distal part of great toe by using a novel flap technique for providing protection in the absence of a nail plate.

\section{Surgical technique and case report}

A 25 years old female patient with bilateral severe onychodystrophy characterized by nail plate thickening and deformation resulting from inadvertent previous nail surgeries visited to our clinic (Figure 1). During the treatment of the patient, the principles of 1975 Declaration of Helsinki were followed. Surgical intervention was performed under local digital bloc anesthesia and Salem's digital tourniquet. Proximal complete nail matrix resection including the underside of the proximal nail fold was performed following total nail plate avulsion. To prevent postoperative nail spike formation, nail matrix horns were ablated by using electrocautery after total surgical resection of nail matrix was completed. Approximately 8-9 mm wide transverse strip distal nail bed resection was performed to replace thin and fragile soft tissue of nail bed with thick and strong tissue of plantar skin. A $5 \mathrm{~mm}$ wide strip of nail bed was left intact proximally between two resection sites for cosmetic reasons (Figure 2A). Proximal nail matrix defect was closed by using the skin of proximal nail fold. The distal nail bed defect was reconstructed by using triple flap technique which was composed of a main central advancement flap containing ventral great toe skin and two side flaps containing nail fold skin of both sides of distal great toe (Figure 2B). The side flaps containing nail fold skin bilaterally were raised to obtain maximum dorsal mobilization of the central advancement flap containing plantar skin of distal part of great toe. Each one of these side flaps was shortened $2 \mathrm{~mm}$ from distal edge of the flap for better coaptation following dorsal transfer of central advancement flap. All the flaps were sutured in their final places after removal of tourniquet and control of bleeding. Operation is completed with wound dressing with slight

Copyright (C) 2021 Aksoy B. This is an Open Access article distributed under the terms of the Creative Commons Attribution-NonCommercial 4.0 International License (http://creativecommons.org/licenses/by-nc/4.0/), permitting all non-commercial use, distribution, and reproduction in any medium, provided the original work is properly cited. 
pressure. The patient was restricted for 14 days and normal activity was allowed after suture removal following this period. The patient healed without any problem and her nail problem was treated successfully. The cosmetic appearance of her first toes was acceptable 8 months after operation (Figure 3). There was no regrowth of nail plates bilaterally. Effective and stable soft tissue coverage of dorsal surface of distal phalanx bone was provided by the thick plantar skin transferred dorsally by using the triple advancement flap method.

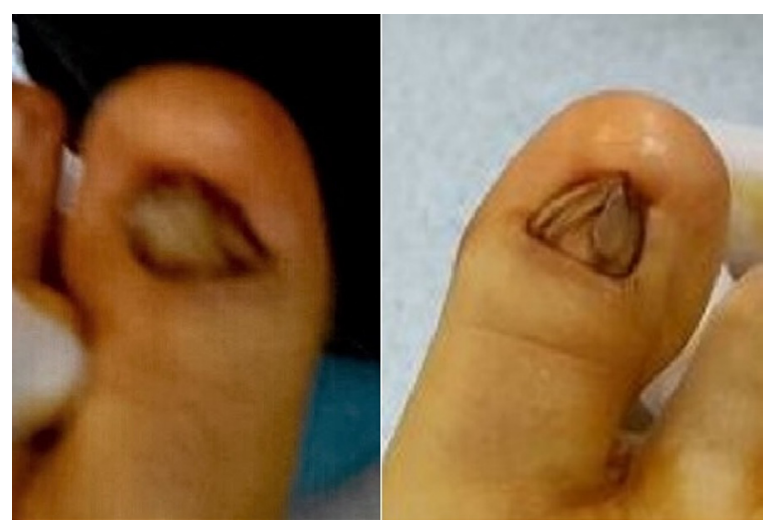

Figure 1. Preoperative picture of severe bilateral nail plate dystrophy involving great toe (A) left, (B) right.
(A)

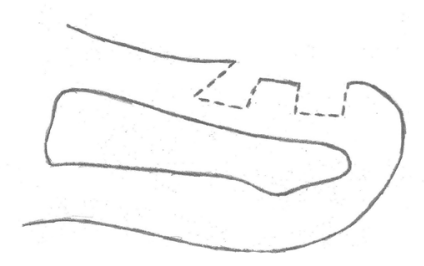

(B)

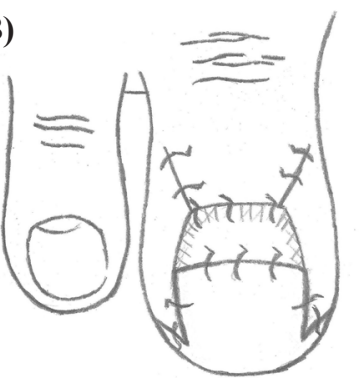

Figure 2. (A) Appearance after proximal and distal nail bed resections. (B) Appearance after reconstruction with triple volar flap.

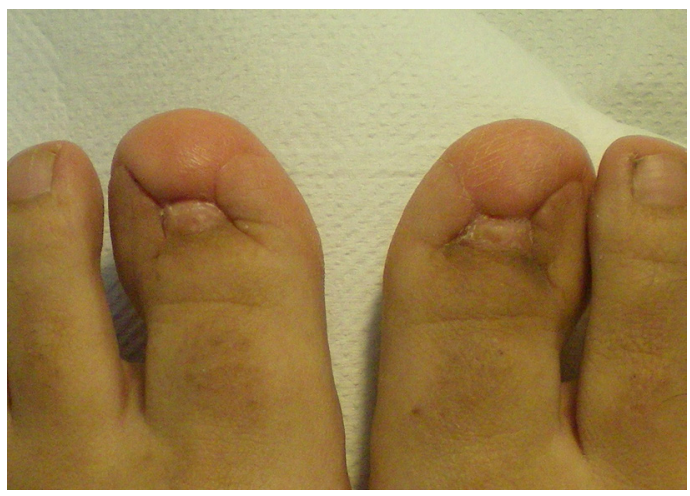

Figure 3. Late postoperative picture of the right and left great toes

\section{Discussion}

Total permanent nail ablation for the treatment of severe nail dystrophies involving the nail plate can be performed by total surgical nail matrix excision and total ablation of nail matrix with electrocautery, chemicals or laser ${ }^{[1-3]}$.

Clinical results of phenol cauterization of nail matrix compares favorably with surgical ablation ${ }^{[1,2]}$. Phenol cauterization results in fewer nail spicule formation and less postoperative pain ${ }^{[1,2]}$. However, addition of bilateral nail matrix horn ablation by using electrocautery will decrease the risk of postoperative spicule formation greatly following surgical total nail matrix excision. Significant cardiotoxicity of phenol due to systemic absorption from dermal application site is a problem especially when patients have cardiac disorders ${ }^{[4]}$. Ten percent sodium hydroxide can also be used to perform total nail matrix destruction to avoid systemic toxic effects of phenol use ${ }^{[3]}$. Chemonecrosis of the surrounding tissues is a potential problem with both phenol and sodium hydroxide nail matrix ablation. Thus, chemical cauterization of the whole nail matrix with significant damage to surrounding soft tissues causes damage to thin soft tissue layer which covers the dorsal surface of the distal phalanx bone. Therefore, scarred nail bed area following recovery from chemical injury will lead to less stable soft tissue coverage for underlying bone. Total chemical nail matrix ablation method also leaves nail bed area unprotected because protective function of nail plate is lost.

Total nail matrix ablation by using electrocautery and lasers also leave dorsal part of distal great toe unprotected because nail plate will never grow again in the absence of nail matrix ${ }^{[2,3]}$.

Surgical resection of nail matrix for total permanent nail ablation may be used for the treatment of difficult nail plate deformities ${ }^{[5]}$. We used modification of the Zadic technique which is an effective method for total surgical matricectomy ${ }^{[3]}$. Effective execution of surgical total nail matrix excision combined with electrosurgical ablation of nail matrix horns bilaterally results in total nail ablation and postoperative nail spike formation is unlikely. Since protective function of nail plate is lost following total nail matrix resection the nail bed area and underlying distal phalanx bone is open to injury. This problem was overcome by replacing thin and fragile nail bed tissue with thick and durable plantar skin through the use of triple flap technique in our case. Triple flap technique is an extension of lateral foldplasty technique that was described previously and used to treat ingrown toe nails ${ }^{[6]}$. Distal nail bed defect associated with resection of subungual exostosis and overlying callus was reconstructed successfully by using a modification of this triple flap technique ${ }^{[7]}$. Pincer nail deformity can be successfully treated by a similar modification of this triple flap technique ${ }^{[8]}$.

Radical nail matrix resection or the Syme procedure involves the radical en bloc resection of the entire nail complex $^{[3]}$. The plantar faciocuteneous flap formed from amputation of the distal half of the terminal phalanx bone is sutured dorsally over the soft tissue defect created 
from the excision of the nail matrix complex for primary wound closure. This surgical method is associated with a success rate of almost $100 \%$ despite the poor cosmetic and functional outcome ${ }^{[3]}$. This method must be reserved for patients with recurrent nail plate deformities that are refractory to repeated treatments of total nail matricectomy.

\section{Conclusion}

Surgical nail ablation followed by plantar skin coverage of dorsal surface of the distal phalanx bone by using triple flap technique is an effective surgical treatment method for the correction of advanced nail plate deformities requiring total nail ablation with a low recurrence rate. Effective and stable soft tissue coverage of dorsal part of distal great toe is provided by this treatment method which replaces thin nail bed tissue with thick plantar skin of the first toe. Cosmetic appearance is satisfactory as a $5 \mathrm{~mm}$ strip of nail bed tissue is left in place. To the best of our knowledge, there is no other surgical technique described for the compensation of loss of protective function of a nail plate following total nail matrix destruction.

\section{Author contributions}

Study concept and design: BA, HMA; Acquisition of data: BA, HMA; Analysis and interpretation: BA, HMA; Study supervision: HMA.

\section{Funding source}

This research received no specific grant from any funding agency in the public, commercial, or not-for-profit sectors.

\section{Conflict of interest}

The authors declare that there is no conflict of interest. This study was poster-presented at the $25^{\text {th }}$ EADV Congress, 28 September -2 October, 2016, Vienna, Austria and at the $6^{\text {th }}$ National Dermatologic Surgery Days, 17 - 20 November, 2016, Ankara, Turkey.

\section{References}

1. Becerro de Bengoa Vallejo R, Losa Iglesias ME, Alou Cervera L, Sevillano Fernández D, Prieto Prieto J. Total nail ablation for onychodystrophy with optimized gauze-phenol application. J Eur Acad Dermatol Venereol 2010; 24(8): 936-42. doi: 10.1111/j.14683083.2010.03569.x.

2. Andrew T, Wallace WA. Nail bed ablation - Excise or cauterise? A controlled study. Br Med J 1979; 1(6177): 1539. doi: 10.1136/bmj.1.6177.1539.

3. Ceilley RI, Collison DW. Matricectomy. J Dermatol Surg Oncol 1992; 18: 728-34. doi: 10.1111/j.15244725.1992.tb02007.x.

4. Li Z, Zhang H, Li SH, Byard RW. Fatal phenol toxicity following attempted tattoo removal. J Forensic Sci 2016; 61(4): 1143-5. doi: 10.1111/1556-4029.13106.

5. Johnson DB Jr, Ceilley RI. A revised technique for ablation of the matrix of a nail. J Dermatol Surg Oncol 1979; 5(8): 642-3. doi: 10.1111/j.1524-4725.1979. tb00738.x.

6. Aksoy B, Aksoy HM, Civas E, Oc B, Atakan N. Lateral foldplasty with or without partial matricectomy for the management of ingrown toenails. Dermatol Surg 2009; 35(3): 462-468. doi: 10.1111/j.1524-4725.2009.01067.x.

7. Aksoy HM, Aksoy B, Balcı M, Üstün H. Onychoclavus due to subungual exostosis: Reconstruction of the resulting defect by triple volar advancement flap: Surgical technique. Turk Klin Tip Etigi Hukuku Tarihi 2011; 31(2): 496-499. doi: 10.5336/medsci.2010-19078.

8. Aksoy B, Aksoy HM. Novel surgical method for pincer nail treatment: Partial matricectomy and triple flap technique. Dermatol Surg 2017; 43(11): 1397-1399. doi: 10.1097/DSS.0000000000001094. 